\title{
História oral de enfermeiras na atenção ao parto e nascimento na Colômbia
}

\author{
Oral history of nurses in the care of labor and birth in Colombia \\ Historia oral de enfermeras en la atención del parto y nacimiento en Colombia
}

\section{Resumo}

Objetivo: Descrever a experiência das enfermeiras no cuidado à mulher no processo de parto e nascimento na Colômbia. Método: Pesquisa qualitativa na História Oral Temática, realizada no período de março de 2018 a dezembro de 2019 por meio de entrevistas com 12 enfermeiras que atuam na área obstétrica nas diferentes instituições de saúde e educação superior de uma cidade ao sul ocidente da Colômbia e analisadas por meio da análise temática de conteúdo. Resultados: Foram encontradas três categorias: O papel, o sentimento e o conhecimento da enfermagem no atendimento à mulher no processo de gestação, parto e pós-parto na Colômbia; as mudanças na atenção à mulher no processo de parto e nascimento, e as estratégias para atenção à gestante, mostrando a trajetória das atividades da enfermeira na atenção à mulher de risco habitual, as leis que impactam na participação direta e o fornecimento de um cuidado integral, sendo um profissional capacitado para atuar na área obstétrica. Conclusão: As enfermeiras identificam fortalezas e fragilidades no cuidado à mulher no ciclo gravídico puerperal e propõem estratégias para o atendimento, além de reconhecerem a importância do uso de boas práticas em prol do parto humanizado.

Palavras-chave: História; Enfermagem; Obstetrícia; Parto humanizado; Colômbia.

\begin{abstract}
Objective: To describe the experience of nurses in caring for women in the labor and birth process in Colombia. Method: Qualitative research on Thematic Oral History, carried out from March 2018 to December 2019 through interviews with 12 nurses who work in the obstetric area in different health and higher education institutions in a city in the south west of Colombia and analyzed through thematic content analysis. Results: Three categories were found: The role, feeling and knowledge of nursing in caring for women in the process of pregnancy, childbirth and postpartum in Colombia; changes in care for women in the process of delivery and birth, and strategies for care for pregnant women, unveiling the trajectory of nurses' activities in the care of women at risk, the laws that impacted their direct participation and the provision of care integral, being a qualified professional to work in the obstetric area. Conclusion: The nurses identify strengths and weaknesses in the care of women in the pregnancy-puerperal cycle and propose strategies for care, in addition to recognizing the importance of using good practices in favor of humanized childbirth.
\end{abstract}

Keywords: History, Nursing; Obstetrics; Humanized delivery; Colombia. 


\begin{abstract}
Resumen
Objetivo: Describir la experiencia de enfermeras en el cuidado de la mujer en proceso de parto y nacimiento en Colombia. Método: Investigación cualitativa con enfoque en Historia Oral temática, realizada en el periodo en el periodo de marzo de 2018 a diciembre de 2019 por medio de entrevistas con 12 enfermeras que actúan en el área obstétrica en las diferentes instituciones de salud y educación superior de una ciudad al sur occidente de Colombia y analizadas por medio de análisis temático de contenido. Resultados: Fueron encontradas tres categorías: El papel, sentimiento y conocimiento de enfermería en la atención de la mujer en proceso de gestación, parto y posparto en Colombia, Cambios en la atención de la mujer en proceso de parto y nacimiento y Estrategias para la atención de la gestante; desvelando la trayectoria de las actividades de la enfermera en la atención a la mujer de bajo riesgo, las leyes que impactaron en su participación directa y en la prestación de un cuidado integral, siendo un profesional capacitado para actuar en el área obstétrica. Conclusión: Las enfermeras identifican fortalezas y debilidades en el cuidado de la mujer en la gestación, parto y puerperio y proponen estrategias para su atención, además de reconocer la importancia del uso de buenas prácticas en pro del parto humanizado.
\end{abstract}

Palabras clave: Historia; Enfermería; Obstetricia; Parto humanizado; Colombia.

\title{
1. Introdução
}

O processo de parto e nascimento coloca a mulher diante de uma série de mudanças fisiológicas e emocionais, que a levam a ter uma vivência singular com necessidade de cuidado integral e individualizado (Watson, 2000). A participação da enfermeira obstetra no cenário do parto pode favorecer o equilíbrio entre o processo fisiológico e as intervenções necessárias, pois este profissional avalia riscos e identifica possíveis complicações, buscando medidas não invasivas antes de recorrer a tecnologias, dessa forma, prestando assistência de forma segura, individualizada e com baixo custo para a instituição (Campos et al, 2016a).

O cuidado é um conceito que abrange múltiplos significados para a ciência, sendo para a enfermagem o principal objeto desta prática. Jean Watson o define como uma relação terapêutica entre duas pessoas, que é subjetiva, porém recíproca (Watson, 2000).

Segundo a Organização Mundial da Saúde (OMS), durante as últimas décadas houve um aumento considerável de práticas que permitem iniciar, regular, acelerar e finalizar o trabalho de parto. Sendo o processo de parto um evento fisioló gico, não deveria sofrer interferências no seu curso, salvo com justificativa, pois toda mulher tem direito a uma experiência de vida significativa e a um parto pautado no respeito aos direitos humanos, sexuais e reprodutivos, além de junto a sua família, ser protagonista deste acontecimento (World Health Organization, 2018).

A atenção ao parto vem experimentando mudanças nas últimas décadas. Durante muito tempo, o parto era atendido na área domiciliar por parteiras em um ambiente privativo e familiar em que a parturiente era o centro do cuidado. Aproximadamente no século XIX, o desenvolvimento tecnológico na saúde influenciou a institucionalização do parto, o declínio da profissão das parteiras e sua participação no cuidado direto à gestante e à parturiente, ficando como atividade exclusiva do corpo médico (Gualda, 2017).

Assim, o parto, que era um momento íntimo e familiar, passou a ser vivenciado na área hospitalar como um ato público em que há separação da mulher de seu ambiente e família. Também evidenciou o uso indiscriminado da tecnologia na realização de intervenções, às vezes desnecessárias, que acabam por gerar eventos adversos para a saúde da mulher e do recém-nascido, tornando o cenário violento e impessoal (Floyd, 2001; Pieszak et al., 2020).

Para tanto, a OMS tem promovido a elaboração de documentos em relação ao parto vaginal, tendo criado recomendações para evitar práticas injustificadas e focar o cuidado na mulher. Em 1996, a Organização publicou um guia prático, citando a enfermeira obstetra como um profissional qualificado, capacitado e valioso para assistir diretamente a gestante de risco habitual e contribuir na humanização (WHO, 2018).

É assim que evidências científicas demonstram que o apoio físico e emocional fornecido à mulher durante o trabalho de parto, seja por uma parteira ou enfermeira, traz benefícios como um parto mais curto, menor uso de medicação e anestesia 
peridural e um parto menos instrumentalizado (WHO, 2018)

Na Colômbia, segundo informe do Fundo de Populações das Nações Unidas (UNFPA), 98\% dos partos são atendidos em instituições hospitalares, uma porcentagem que deveria garantir a saúde do binômio mãe-filho. No entanto, as taxas de mortalidade materna continuam elevadas mesmo que nos últimos anos tenha ocorrido redução. Em 2013, a taxa de morte materna correspondeu a 59 por 100.000 nascidos vivos (Coomeva EPS, 2014), e em 2017, a 51,01 por 100.000 nascidos vivos, por causas que poderiam ter sido evitadas (Colômbia, 2019).

Nesse sentido, com o intuito de reduzir essas taxas, a Colômbia faz parte dos 193 países que compõem a Agenda de Desenvolvimento Sustentável e busca atingir o objetivo três, que é melhorar a saúde de todos(as) em todas as idades, sendo uma das metas, promover a saúde materna pré-natal (Eljach \& Mantilla, 2017), para contribuir para o aumento de nascimentos atendidos por profissionais qualificados, sendo a enfermeira obstétrica capacitada e valorizada pela OMS para prestar esse cuidado em conjunto com uma equipe multiprofissional e reduzir as taxas de mortalidade materna. Para tanto, este estudo teve por objetivo descrever a experiência das enfermeiras na atenção ao parto e nascimento na Colômbia.

\section{Metodologia}

Estudo qualitativo baseado no referencial metodológico descrito por Meihy e Holanda o qual adotou o método história oral temática híbrida, que consiste em centrar a análise na visão dos colaboradores, trabalhando com a experiência deles, (Meihy \& Holanda, 2017), tendo sido conduzido no período de março de 2018-abril de 2019 em Cali, Colômbia, cidade localizada ao sul ocidente do País. Participaram deste estudo 12 enfermeiras que atuam no processo de parto e nascimento nas diferentes instituições de saúde e educação superior no programa de Enfermagem.

Em história oral, as participantes são denominadas de colaboradoras do estudo e fazem referência à presença de duas ou mais delas em uma ou múltiplas entrevistas, em que há uma relação ativa, sendo colaborador tanto quem narra como quem registra, para tanto, o grupo amostral neste método se constrói a partir dos conceitos de Comunidade de Destino, colônia e rede, que são apresentados para fins deste estudo. Assim, todas as enfermeiras que atuam na área obstétrica foram consideradas a Comunidade de Destino. A Colônia é uma fração da Comunidade Destino, composta por enfermeiras que atuam na área da obstetrícia nas diferentes instituições de saúde e de educação superior de uma cidade ao sul occidente da Colômbia, e a Rede é uma subdivisão da colônia que conformou a amostra, composta por 12 enfermeiras que atuam na assistência ao parto e nascimento ou exercem à docência na área da obstetrícia com experiência laboral há mais de dois anos, sendo construída a partir de uma informante chave, enfermeira docente de uma universidade de Cali e a colaboração de um Programa de Saúde Sexual e Reprodutiva da cidade, os quais indicaram os primeiros contatos conforme critérios de inclusão para esta pesquisa.

Os dados foram coletados no período de março-abril de 2019 por meio da entrevista semiestruturada gravada, com o uso de perguntas norteadoras que ajudaram a conduzir o diálogo, possibilitando a interação em relação ao acréscimo de informações de interesse para o estudo. A entrevista foi conduzida pela pesquisadora principal e seguiu três etapas: Pré-entrevista, inicialmente feita por contato pessoal, via WhatsApp, telefone e/ou e-mail. Foram apresentados o projeto, o objetivo do estudo e a participação, e o instrumento de coleta de dados. Em seguida, foi realizada a entrevista no local e data definidos pelo colaborador, que envolveram espaços hospitalares e universitários isolados, garantindo a privacidade, boa iluminação e a ausência de interrupções, sendo registrada a narrativa com uso de um gravador digital, aproximadamente com duração média de uma hora. Para tanto, o grupo amostral foi constituído até alcançar a saturação de dados, levando em consideração as características específicas do estudo proposto, conforme o objetivo, o referencial teórico, além das particularidades dos colaboradores, sendo um processo ativo de reflexão contínua por parte dos pesquisadores (De Souza, 2017).

Na pós-entrevista, deu-se continuidade às etapas de transcrição, textualização, momento caracterizado pela identificação do "Tom vital", recurso que reflete a essência da entrevista, destacando uma frase guia, e, a transcriação dos relatos, construção 
de um novo texto, posteriormente enviado aos colaboradores na versão em língua espanhola, os quais validaram a narrativa, garantindo que seus próprios significados e perspectivas estivessem representados.

As narrativas foram processadas conforme a análise de conteúdo descrita por Bardin, e os trechos, transcritos para a língua portuguesa, seguindo três etapas: pré-análise (leitura flutuante das 12 entrevistas); exploração do material (transformação e organização dos dados em dez Unidades de Registro - UR, agrupadas em três categorias); e tratamento dos resultados, inferência e interpretação (avaliação do material conforme objetivo proposto e sistematização e julgamento dos resultados) (Bardin, 2009). Também foram analisados em relação à história oral híbrida e, cada categoria discutida em base ao cuidado de enfermagem e parto humanizado.

O estudo foi financiado pela Coordenação de Aperfeiçoamento de Pessoal a Nível Superior (CAPES) e aprovado pelo comitê de Ética de uma Universidade Pública ao sul ocidente Colombiano em 2019, com parecer de aprovação No 003-019. Foram devidamente assinados, o Termo de Consentimento Livre e Esclarecido - TCLE e a Carta de Cessão de direitos, além de manter no anonimato a identificação dos colaboradores conforme estabelecido previamente por meio de siglas/letras, como, por exemplo, E1, E2 e assim sucessivamente.

\section{Resultados}

As colaboradoras/enfermeiras tinham entre 26 e 68 anos de idade, oito delas atuando na assistência à mulher no processo de parto e nascimento e quatro na docência no ensino superior da enfermagem. Nove realizaram estudos complementares na área de saúde da mulher, educação e administração. A enfermeira com maior experiência laboral na área tem 45 anos de trabalho e a enfermeira com menor experiência dois anos. A seguir é detalhado o Tom vital das narrativas identificado na pós-entrevista conforme apresentado no Quadro 1.

Quadro 1 - Tom vital das narrativas.

\begin{tabular}{|l|l|}
\hline Enfermeira & \multicolumn{1}{|c|}{ Tom vital } \\
\hline E1 & A integralidade do cuidado foi perdida \\
\hline E2 & As mulheres grávidas são uma bomba-relógio \\
\hline E3 & A cultura é muito respeitada aqui \\
\hline E4 & A atualização constante é essencial na área obstétrica para garantir o bem-estar do binômio \\
\hline E5 & Nós sempre tentamos apagar incêndios \\
\hline E6 & Eu não posso levar a mãe ao passeio da morte, a mãe é uma prioridade \\
\hline E7 & O cuidado é personalizado \\
\hline E8 & A vida me colocou como ponta de lança para fazer coisas que saem do comum \\
\hline E9 & Nós hospitalizamos o parto, fazemos com que a mãe se sinta doente \\
\hline E10 & O cuidado prestado pelo profissional de enfermagem tem sido um cuidado integral, pois além de olhar a \\
\hline E11 & Estamos de frente para o País \\
\hline E12 &
\end{tabular}

Fonte: Autores (2020).

Após extração do Tom vital das narrativas, foi realizada a análise de conteúdo de Bardin, pela qual foram identificadas dez Unidades de Registro (UR), organizadas em três categorias com suas respectivas narrativas.

Na categoria "O papel, o sentimento e o conhecimento da Enfermagem no atendimento à mulher no processo de gestação, parto e pós-parto na Colômbia" as enfermeiras resgatam o papel que têm desenvolvido no transcorrer do tempo e 
identificam as ações que têm contribuído ou não em seu saber e fazer, sendo identificadas seis unidades de registro detalhadas a seguir:

\section{UR1 "O sentir da experiência da enfermeira frente ao cuidado da mulher parturiente"}

Ao narrarem a sua vivência, cinco enfermeiras revelaram sentimentos de gratidão na experiência de cuidar da mulher no processo de parturição, identificando-se como enfermeiras no ato de acompanhar a mulher em todo o seu processo.

É uma experiência bem gratificante, é dar vida a uma pessoa, é colaborar nesse processo de adaptação da vida intrauterina à vida extrauterina, é auxiliar a mãe para que seu recém-nascido tenha (...) um ótimo desenvolvimento durante sua infância (E8).

\section{UR2 "Distanciamento do cuidado direto"}

Nesta UR, foi possível identificar que quatro enfermeiras relacionam como efeito negativo o impacto da tecnologia no cuidado da gestante.

Eu acho que a tecnologia tem influenciado fortemente no cuidado, (...) para mim, a parte da integralidade do cuidado se perdeu pelo mesmo contexto de saúde (...) (E1).

UR 3 "Reconhecem que elas têm capacidade para atender o parto de risco habitual e realizam positivamente o cuidado direto em todo o processo de parturição"

Nos depoimentos, seis enfermeiras reconhecem que têm capacidade para atuar novamente na atenção direta do parto.

Eu acho importante que a gente possa atender o parto de baixo risco, porque permite acompanhar a paciente desde o momento em que ela chegou oito horas atrás, porque já estabeleceu uma relação de empatia e confiança com ela, (...) (E1).

\section{UR 4 "O papel da enfermeira no cuidado à mulher na etapa reprodutiva"}

Nas alusões de oito enfermeiras, foram descritas atividades de cuidado à mulher no processo de parturição, por ressaltarem a importância da educação e orientação para a parturiente e sua família.

É feita educação em saúde para a gestante desde que ingressa; sobre a respiração, os cuidados no pós-parto, os cuidados ao recém-nascido (...) e se acompanha no aleitamento materno (...) (E4).

\section{UR 5 "Percepção da enfermeira frente ao parto humanizado"}

Nas falas, as enfermeiras mencionam o que conhecem ou consideram sobre parto humanizado e relacionam ao acompanhamento, ausência de dor, confidencialidade, parto fisiológico e acolhimento da família.

É tratar que seja normal sem intervenções; que seja uma experiência agradável para a mãe em que se forneça um cuidado adequado, que possa ter seu primeiro contato com seu bebê desde o momento em que nasce (...); se orienta e apoia tanto a paciente como sua família. (E8).

\section{UR 6 "Percepção das enfermeiras na atenção à mulher em processo de parto e nascimento"}

São diversas as ações a serem realizadas pelos profissionais da saúde no trabalho de parto que podem ou não favorecer seu curso natural, porém cinco enfermeiras reconhecem as atividades benéficas como aquelas que podem interferir no seu processo.

Quando o familiar ingressa na sala, é gratificante porque a paciente se sente mais tranquila, ainda mais quando é a mãe dela porque já conheceu seu processo (...) mas não temos uma sala para elas ficarem o tempo todo (E7).

Com relação à posição que elas gostariam de adotar durante seu trabalho de parto, depende dos médicos; às vezes eles falam "assim não"; porém, eu falo pode deixá-la porque em nenhuma parte diz em qual posição a mulher tem que parir (E7).

Nesta categoria intitulada "Transformações na atenção do processo de parto e nascimento" salienta-se as transformações, avanços e/ou retrocessos e destacam-se duas unidades de registro: 


\section{UR 7 "Mudanças na atenção do processo de parto e nascimento"}

Duas colaboradoras mostram a perda do papel da enfermeira na atenção à gestante, sendo um fator a insuficiência do ensino na área de saúde da mulher para o estudante de enfermagem.

Anteriormente, nós identificávamos os fatores de risco e de acordo com isso remetíamos a paciente ao médico ou o controle continuava pela enfermagem; (...) tínhamos participação direta e ativa na gestante de baixo risco (E1).

Na unidade de registro $\boldsymbol{U} \boldsymbol{R} \boldsymbol{8}$ "Políticas de impacto na atenção à saúde da mulher"

Entre os relatos das enfermeiras, foram identificadas as leis que impactam na atenção da gestante.

Quando a Lei 100 foi normatizada através da Resolução 412 (...) ela excluiu novamente o enfermeiro da atenção ao parto, ficando só como responsabilidade do médico (...) mas deu-se conta da necessidade da enfermagem para dar cobertura e porque também garantimos um cuidado integral nesse processo (E11)

E por fim, na categoria "Estratégias para a atenção à gestante" as enfermeiras propõem ações de melhoria a partir das dificuldades percebidas no cuidado à mulher no período gravídico puerperal, ressaltando-se duas unidades de registro:

Na unidade de registro UR 9 "Percepção das dificuldades e facilidades na atenção à gestante"

Onze enfermeiras descreveram as barreiras da atenção à gestante tanto do sistema de saúde como dos profissionais e, a partir disso, também as facilidades. Em relação às dificuldades:

Há dificuldade quando a mulher não recebe uma orientação completa no pré-natal, seja por ter ou não sido assistida ou por falta de educação no trabalho de parto, nesse sentido, são criadas lacunas (E5).

Neste sistema de saúde em que é mais importante a produtividade, é difícil fornecer um cuidado integral que seja humanizado (...) (E11).

\section{Em relação às facilidades as enfermeiras mencionaram:}

Trabalhamos em equipe para prestar uma atenção adequada, eficiente e obter melhores resultados. Também o plano de cuidados é estabelecido pela equipe assistencial, médico, enfermeira e os auxiliares de enfermagem (E8).

\section{UR 10 "Enfermeiras propõem estratégias para melhorar o atendimento à parturiente"}

As 12 enfermeiras propuseram estratégias para melhorar a atenção da gestante conforme a identificação dos limitantes.

Para que uma mulher tenha uma experiência mais positiva de seu parto é no momento em que a gestante entra no prénatal, tudo começa ali, porque esse profissional que está nesse vínculo é quem vai dar as orientações (...) para poder identificar os fatores de risco (E2).

É muito importante que onde seja assistido o parto, mesmo sendo de baixo risco, o pessoal seja capacitado (E4).

\section{Discussão}

A experiência das enfermeiras na atenção ao parto e nascimento na Colômbia foi apresentada em três categorias. No que tange ao papel das enfermeiras, o sentimento e o conhecimento na atenção à mulher no processo de parto e nascimento na Colômbia, nos depoimentos as colaboradoras manifestaram expressões em forma de gratidão ao cuidar da mulher e sua família no processo de parto e nascimento, sentimentos que podem estar ligados a um estado emocional positivo, gerado pela percepção subjetiva das próprias experiências laborais, se manifestando na comunicação, a liderança e a reciprocidade (Jimenez, 2007).

Corroborando com esta pesquisa o estudo desenvolvido numa maternidade pública na cidade de Teresina, estado do Piauí no Brasil em 2016 mostrou que há reciprocidade quando a mulher é cuidada no modelo humanizado, pois os resultados confirmam que as puérperas se sentiram acolhidas e apoiadas quanto a seus direitos, especialmente por ter ocorrido uma relação baseada na comunicação (Da Silva et al., 2017).

Mesmo assim, ainda existem barreiras na criação de uma comunicação efetiva, pois nos relatos deste estudo, as colaboradoras mencionaram o uso inadequado de tecnologia como fator limitante da relação enfermeira paciente, levando, como 
consequência, à perda da humanização e da integralidade do cuidado, também expresso no tom vital da E1 "A Integralidade do cuidado foi perdida".

Há evidências científicas que mostram a melhora dos resultados obstétricos com o uso da tecnologia e algumas práticas, no entanto o uso inadequado tem altas probabilidades de causar dano ao estado emocional, físico e fisiológico da mulher (Leal et al., 2014), podendo, porém, ser adotadas tecnologias leves não invasivas no cuidado, sendo algumas delas, a compreensão, a escuta, o acolhimento e o uso de métodos não farmacológicos para o alívio da dor, que seguem as recomendações da OMS (Cabral et al., 2013), tendo como finalidade mudar o modelo hegemônico para um modelo mais integral que envolva as necessidades e expectativas da mulher e o rol que ela ocupa nesse cenário. (Pereira et al., 2018).

Cabe ressaltar que, neste estudo, as enfermeiras falaram da importância de implementar políticas de humanização na Colômbia que orientem na prestação de um cuidado humano e propiciem um aprofundamento dos profissionais da saúde quanto à atenção da gestante e um empoderamento da mulher na tomada de decisões e na participação no seu processo de parto e nascimento.

No entanto, uma pesquisa feita em 2016 se contrapõe, evidenciando que há desumanização mesmo quando existem políticas, pois os direitos não são respeitados, e os profissionais da saúde sentem-se apoiados pelas instituições ao saber que não serão punidos. Nesse sentido, fazem-se necessários o estabelecimento de punições e a reeducação, incentivando os enfermeiros a prestar um cuidado humanizado (Dos Santos, 2016).

Além da educação nas etapas do processo gravídico puerperal, que contribui para uma experiência de parto e uma maternidade mais significativa (Gomes et al., 2019), a enfermagem desenvolve ações na atenção ao parto, chamadas de boas práticas, que garantem os direitos da mulher e diminui os procedimentos invasivos na procura do resgate do parto natural (Campos et al, 2016b).

Nesse sentido, vale a pena destacar que as enfermeiras da Colômbia conhecem as recomendações da OMS, mas, ainda existem lacunas e preconceitos a respeito de algumas práticas, havendo necessidade de aprofundar e capacitá-las na implementação deste modelo de cuidado.

A Secretaria de Saúde de Cali, na Colômbia, vem se esforçando pelo resgate do parto natural, através da implementação de diferentes ações, como a estratégia Instituições Amigas da Mulher e a Infância (IAMI) sob a estrutura normativa do Ministério de Proteção Social e dos direitos das mulheres e crianças (Colômbia, 2011).

Em relação à categoria transformações na atenção do processo de parto e nascimento, a discussão envolve as ações que a enfermeira vem desenvolvendo através do tempo na área de saúde da mulher, mostrando as mudanças no atendimento, pois as políticas impactam diretamente no cuidado prestado pela enfermeira nas diferentes etapas do ciclo reprodutivo.

No pré-natal, o papel da enfermeira, de acordo com as narrativas das colaboradoras, concentra-se principalmente na educação e na orientação em torno da preparação para o parto, na amamentação e na maternidade, bem como na identificação do nível de risco obstétrico, encaminhando-a para a realização dos respectivos exames. Esses resultados são semelhantes aos encontrados em um estudo feito no Brasil, no qual o pré-natal envolve ações que visam à manutenção, promoção da saúde, prevenção de doenças e detecção precoce de riscos (Duarte, 2012).

Em relação ao parto, as colaboradoras relataram que anteriormente as enfermeiras participavam diretamente do parto de baixo risco, mas na década de 1990 aproximadamente, o parto passou a ser assistido apenas pelo médico, motivo pelo qual as enfermeiras foram relegadas a atividades secundárias.

Segundo elas, a causa principal foi a Lei 100, de 1993, que excluiu a enfermeira da atenção direta ao parto, dando maior poder ao médico, conforme descrito na Resolução 412, de 2000, no capítulo Norma Técnica para Assistência ao Parto (Colômbia, 2000), que também fragmentou seu papel e prejudicou sua formação, razão pela qual o assunto deixou de ser estudado no campo acadêmico, seguindo as disposições da norma. 
Atualmente, pela Resolução 3280, de 2018, a enfermeira especialista em materno-perinatal é autorizada a assistir diretamente o parto fisiológico, sendo a profissional responsável pela identificação de fatores de risco psicossociais, doenças associadas e próprias da gravidez e pela construção de um plano integral do pré-natal e do parto (Colômbia, 2017), faltando ainda uma resolução que regulamente o exercício profissional, como é regulamentado no Brasil, pela Lei do exercício profissional em que é descrita sua atuação. Esta é a Lei 7.498, de 1986, e o Decreto 94.406, de 1987, que afirmam estar a Enfermeira Obstétrica habilitada a assistir a gestante, parturiente e puérpera (Resolução COFEN nº 0479/2015)

Nesse sentido, a OMS, com o objetivo de formar profissionais qualificados no mundo, produz os "Global Standards for the Initial Education of Professional Nurses and Midwives", que promovem para as gestantes e suas famílias uma atenção de qualidade, baseada nas suas necessidades e entornos (WHO, 2009). Sendo relevante vincular enfermeiras obstetras aos serviços de saúde na criação de programas estruturados de educação continuada e assim mudar o modelo hegemônico ainda presente no cuidado à mulher.

E por fim, no que se refere às estratégias para a atenção à gestante, aborda propostas feitas pelas enfermeiras, que buscam contribuir para um melhor atendimento à mulher no período gravídico-puerperal, levando em consideração as dificuldades e facilidades que elas percebem na atenção. Uma das dificuldades mencionadas é a falta de educação no pré-natal, fator que pode desfavorecer o processo natural do parto.

Esses resultados são semelhantes aos encontrados num estudo que avaliou a satisfação das gestantes na assistência ao pré-natal, em que as mulheres se sentirem insatisfeitas em relação à educação em saúde, fator que contribui à tomada de decisões erradas pelas mulheres, com possibilidade de alterar o curso do processo de parto (Guerreiro et al., 2012).

Outra dificuldade mencionada se relaciona aos fatores que limitam o atendimento da gestante como prioridade, destacando o Sistema de Saúde e, principalmente, as barreiras impostas na área administrativa, o que se relaciona com os convênios entre as Empresas Promotoras em Saúde EPS e as Instituições Prestadoras de Saúde IPS na qualidade da atenção medida em produtividade na Colômbia.

Essas barreiras são semelhantes às encontradas em estudos conduzidos em outros países como Chile e Argentina, onde a área administrativa se esquece dos direitos das usuárias, sendo burocráticos no atendimento (Landini et al., 2015; Poffald et al., 2013).

Apesar das limitações e barreiras do sistema organizacional de saúde, não apenas a Colômbia, mas também alguns países se esforçam para melhorar o acesso e a qualidade dos serviços, principalmente aqueles de ordem materno-perinatal, tendo em conta que é um indicador fundamental de impacto social.

No caso do Brasil, o Ministério da Saúde tem lançado programas de educação continuada para as enfermeiras obstetras, políticas e ações que visam ampliar o acesso, melhorar a qualidade da atenção pré-natal, parto e puerpério, além de garantir o atendimento da criança até os 24 meses, como no caso do programa Rede Cegonha (Cavalcanti et al., 2013).

Neste estudo, as enfermeiras propuseram estratégias de melhoria para o atendimento da mulher no período gravídico puerperal, destacando a implementação das recomendações preconizadas pela OMS em direção ao modelo de parto humanizado.

Nesse sentido, diferentes evidências científicas mostram que o modelo instaurado pela OMS põe a mulher e sua família como sujeitos principais do cuidado, envolve características de sensibilidade, respeito e dignidade com a finalidade de gerar autonomia na mulher, garantir segurança nos procedimentos, beneficiar e mudar o modelo biomédico que vem sendo utilizado (Damaceno, 2015).

Cabe destacar que entre as estratégias, as colaboradoras mencionaram a importância do treinamento e a atualização contínua dos profissionais e das instituições, apontado também no tom vital da E4 "A atualização constante é essencial na área obstétrica para garantir o bem-estar do binômio" ratifica a necessidade dos profissionais serem capacitados na busca de um atendimento melhor. Por outro lado, propõem o aconselhamento pré-concepcional pela enfermeira para a mulher e seu 
companheiro, consulta que permite promover a saúde para o desenvolvimento de uma gravidez saudável, a identificação de riscos a prevenção de agravos na gravidez e planejar os cuidados no intuito de melhorar a saúde materna, sendo evidenciado em diferentes estudos (Borges et al., 2016).

Na Colômbia, a atenção pré-concepcional foi estabelecida pela Resolução 3280, de 2018, e pela Resolução no 276 de 2019, com prazo de implementação de três anos a partir do $1^{\circ}$ de janeiro de 2020 (Resolução $n^{\circ}$ 000276/2019), o que desfavorece a identificação e o controle de riscos potenciais que impactam as taxas de morbimortalidade materna e infantil.

\section{Considerações Finais}

Este estudo possibilitou atingir ao objetivo proposto, pois a história oral temática como método permitiu aprofundar na vivência das enfermeiras na realidade em que se desenvolvem na atenção à mulher no período gravídico puerperal, além de revelar informação atual e histórica da Enfermagem no atendimento desta população, bem como a posição das enfermeiras na assistência à gestante de risco habitual, e o impacto que tem causado ao Sistema de Saúde da Colômbia diante do papel desempenhado por elas na atenção direta do parto.

O tema é relevante e atual, na medida que aponta as conquistas das enfermeiras obstétricas na Colômbia e os retrocessos que vêm ancorados no paradigma biomédico e medicalizante e respaldados pela legislação do país. Aponta a necessidade de investimento político da Enfermagem para melhorar o processo de autonomia profissional [políticas públicas de formação/qualificação e autonomia profissional].

Adicionalmente, contribui para que a enfermeira reflita sobre seu agir na área obstétrica, pois conhecer o papel que ela desenvolve no cuidado à gestante evidencia a capacidade e o conhecimento que elas têm para atuar com competência na atenção direta ao parto, ao mesmo tempo em que fortalece o âmbito investigativo, oferecendo uma visão da situação atual do país e as barreiras que podem ser melhoradas na saúde materna.

Ressalta-se que as enfermeiras da Colômbia estão cientes da necessidade de gerar mudanças no atendimento da parturiente, com o uso das boas práticas, para tanto, elas devem ter maior autonomia em sua prática e na construção de uma resolução que regulamente esses procedimentos para guiar seu trabalho na assistência, além de gerar movimentos construtivos que tornem seu conhecimento e agir visível.

Como limitação deste estudo, pode-se citar a escassa literatura sobre o cuidado de Enfermagem na área obstétrica na Colômbia e o modelo de assistência estar ainda pautado no modelo biomédico, sendo essa limitação uma base para compreender o problema de pesquisa e apontar possíveis ações de melhoria, portanto, considera-se importante para estudos futuros, resgatar as experiências de mulheres que vivenciaram o processo de parto e nascimento, a fim de identificar aspectos que possam transformar dito modelo, e a participação do profissional baseado nas necessidades e expectativas da população.

\section{Referências}

Borges, A. L. V., Santos, O. A. D., Nascimento, N. D. C., Chofakian, C. B. D. N., \& Gomes-Sponholz, F. A. (2016). Preparo pré-concepcional entre mulheres brasileiras e a relação com o planejamento da gravidez. Revista da Escola de Enfermagem da USP, 50(2), 208-216. http://www.scielo.br/scielo.php?pid=S0080$62342016000200208 \&$ script=sci_arttext\&tlng=pt

Bardin, L. (2009). Análise de conteúdo. Edições, 70(3), 5-118.

Cabral, F. B., Hirt, L. M., der Sand, V., \& Pacheco, I. C. (2013). Atendimento pré-natal na ótica de puérperas: da medicalização à fragmentação do cuidado. Revista da Escola de Enfermagem da USP, 47(2), 281-287. Recuperado em: http://www.scielo.br/pdf/reeusp/v47n2/02.pdf

Campos, B. C. V., Pereira, É. P., Medeiros, G. A. D. \& Pereira, E. P. (2016a). Perfil da assistência hospitalar por enfermeiras residentes em obstetrícia em Brasília, Distrito Federal. Comun. ciênc. saúde, 27(4), 291-300.

Campos, N. F., Maximin D. A. F. M., Virgínio N, \& de Souto, C. G. V (2016b). A importância da Enfermagem no parto natural humanizado: uma revisão integrativa. Rev Cienc Saúde Nova Esperança, 14 (1): 47-58. http://revistanovaesperanca.com.br/index.php/revistane/article/view/76 
Cavalcanti, P. C. D. S., Gurgel Junior, G. D., Vaconcelos, A. L. R. D., \& Guerrero, A. V. P. (2013). Um modelo lógico da Rede Cegonha. Physis: revista de saúde coletiva, 23, 1297-1316. http://www.scielo.br/scielo.php?pid=S0103-73312013000400014\&script=sci_abstract\&tlng =pt

Colômbia (2000). Ministerio de salud. Dirección general de promoción y prevención. Norma técnica para la atención del parto. Bogotá: Ministerio de salud, 2000. https://www.minsalud.gov.co/sites/rid/Lists/BibliotecaDigital/RIDE/VS/PP/3Atencion\%20del \%20Parto.pdf

Colômbia (2011). Ministerio de Protección Social. UNICEF. Documento final de trabajo: Lineamientos IAMI. Criterios globales para el cumplimiento de los 10 pasos de la Estrategia Instituciones Amigas de la Mujer y la Infancia y formulario de autoapreciación para instituciones de salud hospitalarias y ambulatorias. Bogotá: Ministerio de Protección Social-Unicef. 141 p. https://www.minsalud.gov.co/sites/rid/Lists/BibliotecaDigital/RIDE/VS/PP/SNA/lineamiento s-ami2011.pdf

Colômbia (2017). Subsecretaría de servicios de salud y aseguramiento. Dirección de provisión de servicios de salud grupo materno perinatal. Ruta de atención integral en salud: materno perinatal 2017. Bogotá: Subsecretaría de servicios de salud y aseguramiento, 2017. https://grupodinamizador.jimdo.com/app/download/9963570370/RIAS+MATERNO+PERIN ATAL.pdf?t=1519830389

Colômbia. (2019). Ministerio de salud y protección social. Dirección de epidemiología y demografía. Análisis de Situación de Salud (ASIS) Colombia, 2018. Bogotá: [s. n.]. 273p.

Coomeva EPS (2014, 10 de noviembre). Preocupan las cifras de mortalidad materna en Colombia: la Mortalidad Materna puede prevenirse planeando los embarazos y acudiendo a los controles médicos establecidos por el sistema de salud para esta etapa [Comunicado de prensa]. http://eps.coomeva.com.co/descargar.php?id=10439.

Da Silva, I. A., Andrade, É. W. O. F., De Morais, F. F., Silva, R. S. D. S., \& Oliveira, L. S. (2017). Percepção das puérperas acerca da assistência de enfermagem no parto humanizado. Revista Uningá, 53(2), 37-43. http://revista.uninga.br/index.php/uninga/article/view/1440/1057

Damaceno D. C. (2015). A importância do parto humanizado: atenção da equipe de Enfermagem. FACIDER-Revista Científica, (7), 1-13. http://www.seicesucol.edu.br/revista/index.php/facider/article/view/132/167

De Souza, M. C. (2017). Amostragem e saturação em pesquisa qualitativa: consensos e controvérsias. Revista pesquisa qualitativa, 5(7), 1-12. Recuperado em: https://editora.sepq.org.br/rpq/article/view/82

Dos Santos, M. B. B. (2016). Violência obstétrica: a violação aos direitos da parturiente e a desumanização do parto. Revista de Direito UNIFACEX, 7(1), 123. https://periodicos.unifacex.com.br/direito/article/view/869/285

Duarte, S. J. H. (2012). Motivos que levam as gestantes a fazerem o pré-natal: um estudo das representações sociais. Ciencia y Enfermeria, 18(2), 75-82. https://scielo.conicyt.cl/pdf/cienf/v18n2/art_08.pdf

Eljach, G. \& Mantilla, J.H. (Agosto 8 de 2017). Proyecto de ley número 063 de 2017. Por medio de la cual se establecen los derechos de la mujer en trabajo de parto, parto y postparto y se dictan otras disposiciones o ley de parto humanizado. https://www.asivamosensalud.org/politicas-publicas/normatividad-proyectosde-ley/derechos/proyecto-de-ley-063-de-2017-ley-de-parto

Floyd, R.D. (2001). The Technocratic, Humanistic, and Holistic Paradigms of Childbirth. International Journal of Gynecology and Obstetrics. Int J Gynaecol Obstet. 2001, 75(1), S5-S23. https://www.researchgate.net/publication/230669902_The_Technocratic_Humanistic_and_Holistic_Models_of_Birth

Gomes C. B. D. A., Dias, R. D. S., Silva, W. G. B., Pacheco, M. A. B., Sousa, F. G. M. D. \& Loyola, C. M. D. (2019). Consulta de enfermagem no pré-natal: narrativas de gestantes e enfermeiras. Texto \& Contexto-Enfermagem, 28, 01-15. https://www.scielo.br/scielo.php?pid=S0104$07072019000100320 \&$ script=sci_arttext\&tlng=pt

Gualda D.M.R., Campos E.A., Praça N.S., Salim N.R. \& Soares G.C.F. (2017). Nascimento: Perspectivas Antropológicas. Ícone.

Guerreiro, E. M., Rodrigues, D. P., Silveira, M. A. M. D., \& Lucena, N. B. F. D. (2012). O cuidado pré-natal na atenção básica de saúde sob o olhar de gestantes e enfermeiros. Revista Mineira de Enfermagem, 16(3), 315-323. https://docplayer.com.br/9544728-O-cuidado-pre-natal-na-atencao-basica-desaude-soboolharde-gestantes-e-enfermeiros.html

Jimenez, M. A. (2007). Factores del clima organizacional influyentes en la satisfacción laboral de enfermería, concerniente a los cuidados intensivos neonatales del Hospital Nacional de Niños, 2004. Enfermería en Costa Rica, 28(1), 12-19. https://www.binasss.sa.cr/revistas/enfermeria/v28n1/art3.pdf

Landini, F. P., González V., Vanina B., Bianqui, V., Logiovine S., Vázquez, J. \& Viudes, S. (2015). Accesibilidad en el ámbito de la salud materna de mujeres rurales de tres localidades del norte argentino. Saúde e Sociedade, 24(4), 1151-1163. https://www.scielo.br/scielo.php?pid=S0104$12902015000401151 \&$ script=sci_abstract\&tln g=es

Leal, M. D. C., Pereira, A. P. E., Domingues, R. M. S. M., Filha, M. M. T., Dias, M. A. B., Nakamura-Pereira, M., \& Gama, S. G. N. D. (2014). Intervenções obstétricas durante o trabalho de parto e parto em mulheres brasileiras de risco habitual. Cadernos de Saúde Pública, 30, S17-S32. https://www.scielosp.org/pdf/csp/2014.v30suppl1/S17-S32/pt

Meihy J.C.S. \& Holanda F. (2017). História Oral: Como Fazer Pensar. Pinsky Ltda.

Pereira, S. B., Diaz, C. M. G., Backes, M. T. S., Ferreira, C. L. D. L. \& Backes, D. S. (2018). Boas práticas de atenção ao parto e ao nascimento na perspectiva de profissionais de saúde. Revista Brasileira de Enfermagem, 71, 1313-1319. https://www.scielo.br/j/reben/a/XYksDZmcHxdFTppBV87bxrn/?lang=pt

Pieszak, G. M., Gomes, G. C. \& Rodrigues, A. P. (2020). Fatores que interferem no processo de parto e nascimento: revisão integrativa da literatura. Research, Society and Development, 9(7), e638974470-e638974470. https://www.rsdjournal.org/index.php/rsd/article/view/4470 
Research, Society and Development, v. 10, n. 10, e533101019059, 2021

(CC BY 4.0) | ISSN 2525-3409 | DOI: http://dx.doi.org/10.33448/rsd-v10i10.19059

Poffald, L., Hirmas, M., Aguilera, X., Vega, J., González, M. J. \& Sanhueza, G. (2013). Barreras y facilitadores para el control prenatal en adolescentes: resultados de un estudio cualitativo en Chile. Salud Pública de México, 55(6), 572-579. https://www.scielosp.org/scielo.php?pid=S0036$36342013001000006 \&$ script=sci_arttext\&tl ng=es

Resolução COFEN no 0479/2015 - Estabelece os critérios para registro de títulos de Enfermeiro Obstetra e Obstetriz no âmbito do Sistema Cofen/Conselhos Regionais de Enfermagem, e dá outras providência. 2015. Recuperado em: http://www.cofen.gov.br/resolução-cofen-no-4792015_30971.html

Resolución nº 000276/2019 - Por la cual se modifica la Resolución 3280 de 2018. 2019. https://www.minsalud.gov.co/sites/rid/Lists/BibliotecaDigital/RIDE/DE/ DIJ/resolucion-276-de-2019.pd

Watson, J. (2002). Caring science and the science of unitary human beings: a transtheoretical discourse for nursing knowledge development. Journal of advanced nursing, 37(5), 452-461. Recuperado em: https://pdfs.semanticscholar.org/d35d/4d9205bfdb0f9ed32d995821daaaad8ea4c7.pdf

World Health Organization (WHO). (2009). Global standards for the initial education of professional nurses and midwives. WHO. 40 p. Recuperado em: https://www.who.int/hrh/nursing_midwifery/hrh_global_standards_education.pdf

World Health Organization (WHO). (2018). WHO recommendations: intrapartum care for a positive childbirth experience. WHO. 210 p. Recuperado em: http://iris.paho.org/xmlui/bitstream/handle/123456789/51552/9789275321027_spa.pdf?seq uence=1\&isAllowed= 